\title{
Frequency of Temporomandibular Joint Ankylosis in Various Age Groups with Reference to Etiology
}

Mohammad Ali Hossain ${ }^{*}$

Syed Adnan Ali Shah ${ }^{2}$

Rajat Sanker Roy Biswas ${ }^{3}$

'Department of Oral and Maxillofacial Surgery Chattagram International Dental College and Hospital Chittagong, Bangladesh.

${ }^{2}$ Director, Maxillofacial Division

University of Manitoba

Manitoba, Canada.

${ }^{3}$ Department of Medicine

Chattagram International Dental College and Hospital

Chittagong, Bangladesh.
*Correspondence to:

\section{Dr. Mohammad Ali Hossain}

Assistant Professor

Department of Oral and Maxillofacial Surgery Chattagram International Dental College and Hospital Chittagong, Bangladesh.

Mobile: +8801914960351

E-mail:hossyne@hotmail.com

\begin{abstract}
Introduction: Temporomandibular joint ankylosis is a gradually developing condition manifested by mandibular hypomobility. Limitation of mouth opening can be caused by bony or fibrous ankylosis of the temporomandibular joint as sequel to trauma, infection, and autoimmune diseases. The purpose of this study was to determine the frequency of temporomandibular joint ankylosis in various age groups and to see the etiology of temporomandibular joint ankylosis. Materials \& methods: This descriptive study study was carried out at the department of Oral and Maxillofacial surgery, de'Montmorency College of Dentistry / Punjab Dental Hospital, Lahore from September 2004 to August 2005 prospectively. A total 60 patients of various ages having TMJ ankylosis were included. Patients presented with TMJ ankylosis were assessed with detailed history and physical examination. Basic investigations including conventional radiographs and orthopentomogram were undertaken for every patient. Results: Females predominate with 35 (58.30\%). Age ranged from 4 to 36 years and 11-20 years $(60 \%)$ group predominating. In unilateral cases, left side involvement was more common in both males and females. TMJ ankylosis was post traumatic in $47(78.3 \%)$ cases followed by infection $7(11.7 \%)$ while birth trauma $2(3.3 \%)$ was rare. RTA was seen to the most frequent etiological factor followed by the fall. Conclusions: Temporomandibular joint ankylosis is a preventive entity if posttraumatic rehabilitation is instituted early and properly.
\end{abstract}

Key words: Temporomandibular joint; ankylosis; trauma.

\section{INTRODUCTION}

Temporomandibular joint is bilateral, diarthrodial joint in maxillofacial region. The joint is formed by the bony articulation of mandibular condyle and the temporal bone'.

Temporomandibular joint (TMJ) is the part of the stomato-gnathic system. Any factor influencing this joint will affect the function of TMJ, resulting into hypo or hypermobility ${ }^{2}$. The factors influencing the TMJ are numerous but one condition i.e. TMJ ankylosis was taken into consideration in this study.

Temporomandibular joint ankylosis is an intracapsular union of the disc condyle complex to the temporal articular surface that including fibrous adhesion or bony fusion between condyle of mandible and glenoid fossa and articular eminence of squamous temporal bone ${ }^{3}$. It is true joint pathology with bony mass or fibrous tissue, which replaces the normal articulation ${ }^{4}$.

TMJ ankylosis is an affliction that causes untold misery to many patients' particularly young adults. TMJ ankylosis is a very distressing structural condition that denies the victim the benefit of normal diet and opportunities in career that require normal speech ability. It also causes severe facial disfigurement that aggravates psychological stress 5 . 
Ankylosis of TMJ may be classified according to location (intra articular or extra articular), type of tissue involved (bony, fibrous or fibro osseous) or extent of fusion (complete or incomplete $)^{6}$. TMJ ankylosis is also distinguished into true and false ankylosis. In the first case the damaging process occurs intra-articularly by adhesion of the mandibular condyle with the glenoid fossa. In the second case, joint hypo-mobility is due to extra-articular causes among which hyperplasia of coronoid process, untreated fracture of zygomatic arch, surgery- or radiation-induced fibrosis of the temporal muscle and neurological or psychic disorder ${ }^{6}$. Functionally, mandibular mobility (opening, lateral and protrusive) is restricted in both kinds of ankylosis?

Pathogenesis can be distinguished into primary when the pathological process affects TMJ directly, as in the case of septic arthritis, or rheumatic disease and secondary, as in traumatic injury, which is the most common cause of ankylosis. Such injuries may cause intra-capsular condylar fractures, heamarthrosis and secondary hematoma organizing with ensuing ankylotic block formation ${ }^{8}$.

This ailment is caused by various etiological factors including facial trauma (31-98\%), local and systemic infection (10-40\%) congenital as well as neoplasm in TMJ area'. Road traffic accident and falls are considered to be major causes of TMJ ankylosis in children and documented as major etiological factor in $86 \%$ cases $^{10}$. Chandra and Dave reported $67.8 \%$ associated with trauma and $17 \%$ with infection ${ }^{11}$. Kim Su-Gwan found trauma $(85.7 \%)$ was the major cause of unilateral TMJ ankylosis in adult patient ${ }^{5}$.

Post traumatic TMJ ankylosis is not often encountered in the west, though it is the major factor of TMJ ankylosis not only in Pakistan but also in the whole subcontinent ${ }^{11}$. In Pakistan, the main cause was trauma (95.7\%), while infection and neoplasm was rare. In $3.6 \%$ of cases, etiology was not established ${ }^{12}$.

TMJ ankylosis occurs in both children and adult. Early ankylosis of TMJ in children can be a deterrent to normal mandibular growth ${ }^{8}$. Impairment of speech, difficulty with mastication, poor oral hygiene, disturbances of facial and mandibular growth, malocclusion, and acute compromise of airway invariably present unique challenge to surgeon ${ }^{13}$.

Preoperative evaluation for diagnosis usually consists of careful history, physical and radiological examination. The radiographic examination is important at final diagnosis that includes orthopentomogram, plain lateral radiograph and tomogram ${ }^{14}$. Computed tomography examination is of additional value in delineating the extent of pathology and its relationship to surrounding vital structures. Lateral and posteroanterior cephalogram are also drawn to determine extent of facial deformity and its correction ${ }^{15-16}$.

Regardless of age of the patient surgical intervention is the treatment of choice. Three basic techniques have been developed for surgical correction of TMJ ankylosis that are gap arthroplasty, interpositional arthroplasty and joint reconstruction with total joint prosthesis ${ }^{17-18}$.
Recently simultaneous gap arthroplasty and distraction osteogenesis for the correction of mandibular deformity in the management of TMJ ankylosis in children is proposed ${ }^{19}$. The rehabilitation procedure includes the postoperative mouth opening, orthodontic treatment and orthognathic surgery for the correction of the dentofacial deformities ${ }^{2}$.

The incidence of TMJ ankylosis is declining in Europe and North America, partly as a result of better, earlier management of condylar fractures and partly because the use of antibiotics which have reduced persistence and recurrence of infection. TMJ ankylosis is relatively common in developing countries like Pakistan mainly resulting from childhood trauma ${ }^{20}$. Parents either ignore their children from the after effect of trauma or they are not properly managed in hospitals at the time of injury ${ }^{17}$.

Since TMJ ankylosis goes unnoticed for long period of time, early diagnosis and better management can help to reduce morbidity of TMJ ankylosis. The aim of this study is to observe whether TMJ ankylosis is more common in Pakistani population compare to western countries. Furthermore, this study may provide in identifying etiological factors, which may increase the awareness about the problem.

\section{MATERIALS \& METHODS}

This prospective descriptive study was carried out in the Department of Oral \& Maxillofacial Surgery, Punjab Dental Hospital, a tertiary dental health care center of de'Montmorency College of Dentistry, Lahore, Pakistan between September 2004 and August 2005. Sixty patients with TMJ ankylosis were enrolled via non-probability purposive sampling. Diagnosed cases of true ankylosis of either gender were included in the study. Patients with re-ankylosis, other forms of TMJ ankylosis including false types and terminally ill and immunocompromised patients were excluded.

A standardized structured data collection instrument used to collect necessary information. A detailed history of the patients was taken and meticulous clinical examination was performed on all the patients presented with TMJ ankylosis. For each patient, a number of variables were recorded including their demographic data, mouth opening at presentation, size and symmetry of mandible, sleep apnea, age at injury and presentation, duration of ankylosis and treatment received during trauma. All patients were questioned concerning facial trauma and other causes. Examination included TMJ examination, occlusal relationship, maximum interincisal opening, excursion of mandible and deviation. Standard panoramic view (orthopentomogram) was obtained as routine for all study subjects. Lateral cephalometric images were taken to assess hypopharynx in relation to sleep apnea and facial profile. Axial and coronal sections of computed tomography of the TMJ were also advised to few patients to reach conclusion. Definitive diagnosis of TMJ ankylosis was established with the aid of clinical and radiographic findings. Patterns of TMJ ankylosis were determined according to the Topazian classification as follows: 
Stage I: Ankylotic bone limited to the condylar process.

Stage II: Ankylotic bone extending to the sigmoid notch.

Stage III:Ankylotic bone extending to the coronoid process.

All data were entered in SPSS 10 version. Frequency distribution for the entire variables was worked out and results were analyzed accordingly. The data was presented as proportion and percentage. Important findings were presented in graphical form.

\section{RESULTS}

Among the study population females were majority with $58.30 \%$. Table 1 shows the age distribution where 11-20 group predominates in both males and females. Stage I ankylosis comprised the highest proportion (45\%) followed by stage II $(36.7 \%)$ and stage III (5\%). In relation with site of involvement, in males 11 were on left side, 8 on right and 6 bilateral. In females, 18 were on left side, 12 on right and 5 bilateral. Among bilateral ankylosis, 10 cases presented with $\mathrm{h} / \mathrm{o}$ trauma. The presentations of TMJ ankylosis asymmetry of face $76.6 \%$ (46) and deviation of mandible while opening $73.3 \%$ (44) and pre-auricular bulging 53.3\% (32) cases. TMJ ankylosis was found to be multifactorial, however trauma $(78.3 \%)$ and infection $(11.7 \%)$ were predominant causative factors followed by systemic disease, like typhoid fever $(3.3 \%)$ and unknown $(3.3 \%)$. Trauma was main etiological factor in both male and female; where RTA $(38.3 \%)$ and fall $(31.3 \%)$ were majority followed by animal (donkey) kick (6.7\%). Females were found to be more prone to fall $(51 \%)$, whereas males were more to RTA $(52 \%)$.

Table 1: Age distribution $(n=60)$

\begin{tabular}{lccc} 
Age & Male & Female & Total \\
$1-10$ & 8 & 7 & 15 \\
$11-20$ & 13 & 25 & 38 \\
$21-30$ & 4 & 2 & 6 \\
$>31$ & 0 & 1 & 1 \\
\hline
\end{tabular}

\section{DISCUSSION}

Knowledge of the etiology of TMJ ankylosis has not progressed to the same extent as surgical methods for treating the condition and one of the reasons attributable to it can be the range of differences in etiological variables. Our study is also hampered by same problem as time lapse is often a barrier to accurate designation of etiology and lack of information given

by patients. From literature review, it was found that onset of TMJ ankylosis usually occur in children under 10 years. In our study, the mean age at which the disease occurred was 7.07 years.

The frequency of TMJ ankylosis was highest in second decade followed by first decade in our study. It may be that injury to TMJ in growing population i.e. 1-10 years remains unreported due to lack of diagnostic facilities and awareness in the less privileged rural areas subsequently leading to ankylosis.
Females constitute majority of our case series and male:female ratio is 1:1.5. The average age of occurrence for trauma patient was nine in contrast with an average of eleven years for all others. Nineteen out of the 25 patients with ankylosis associated with trauma were male and twenty seven out of the 35 patients were female. This is in contrast with those who suffered from ankylosis from other causes. This is probably due to the difference in playing activities of boys and girls.

The present study carried out at Punjab Dental Hospital has also revealed that trauma is the major cause $(78.3 \%)$ of TMJ ankylosis. Another study in Pakistan, trauma was $70.6 \%$ where as infection was $7.8 \%{ }^{20}$. The high incidence of TMJ ankylosis in Pakistan is because of delay in treatment of intracapsular condylar fracture as a result of:

1. Parents either ignore their children from the after effects of facial trauma or there is lack of awareness regarding post condylar injury complications.

2. Non-availability of surgical expertise in remote area.

3. Fear of pain and consequent prolonged immobilization i.e., IMF of injured TMJ eventually leads to ankylosis.

Road traffic accidents and falls are considered to be the major causes of TMJ ankylosis in children. Falls accounted for 19 patients, 23 were the victim of RTA and 4 of the cases were due to animal kick to the face. Most of traumatic cases received conservative treatment $(41.7 \%)$ at time of injury while remaining cases got long immobilization of jaws and no treatment. Post traumatic physiotherapy is mandatory in childhood mandibular injury to prevent ankylosis.

The relative frequency of falls in our female population (16 cases) remains high compared to male population. This can be attributed to the more home bound nature of female population in Pakistan which makes them less prone to be associated with RTA. Local kite flying tradition and festivities like basant have led to reckless accidents caused by falls from the roof tops while involving the different acts associated with kite flying. Moreover, the unprotected nature of roof tops with a dearth of boundary wall in many localities has resulted in falls. The unilateral and bilateral ratio was $2: 1$. In bilateral ankylosis( $(n=11), 9$ patients were presented with sleep apnea.

According to Qudah et al trauma (91.2\%) was major etiological factor $^{21}$. In the current study, the etiology for the 47 cases examined was all traumatic in nature. Padget observed that frequency of infection as etiologic agent ranges from $44 \%$ to 68 $\%^{18}$. Topazian found that infection was the etiology of TMJ ankylosis in as many as $68 \%$ cases. There were only 7 cases in our series where the problem was the secondary to infection in TMJ areas. We didn't come across any case secondary to rheumatoid disease.

Symptoms are difficult to assign precisely with specific causes because frequently years lapse between the onset of disability and the time history of illness is elicited. The clinician then must deal with memories which may span a decade or more. The problem of time lapse probably has led to the assignment of descriptive terms rather than to more concise diagnose. A careful history, however, often will disclose information which makes assignments of cause easy. Hospital records, if admission was required may give helpful information. 
Traumatic lesions of the TMJ often are overlooked as they can apparently occur with relatively little pain. In 2 cases, diagnosis could not be established. The entity was considered secondary to some missed trauma.

On the basis of present study, it is evident that condylar injuries are overlooked after in immediate post traumatic period. All those cases with common pattern of injury or disturbance to the TMJ must be managed keeping ankylosis as a possible post traumatic complication. This include, direct or indirect blow to the TMJ area, fall causing impact to the chin, scratching and tearing of the internal structure to the joint and dislocating forces.

\section{CONCLUSION}

Trauma is the major cause of TMJ ankylosis in Pakistan. A proper screening of maxillofacial injuries especially condylar injuries and referral to maxillofacial centre is recommended to ensure proper evaluation of etiological factors. Post injury physiotherapy and regular follow up is necessary to prevent TMJ ankylosis.

\section{DISCLOSURE}

All the authors declared no competing interest.

\section{REFERENCES}

1. Rees LA. The structure \& function of mandibular joint. Br Dent J 1954; 96: 125-133.

2. Anwar J. Rehabilitation techniques for post-surgical temporomandibular joint hypomobility. AFID Dental Journal 1987; 6-11.

3. Long X, Li X, Cheng Y, Yang X, Qin L. Preservation of disc for treatment of traumatic temporomandibular joint ankylosis. J Oral Maxillofac Surg 2005;63:897-902.

4. Salin PC. New perspective in the management of craniomandibular ankylosis. Int J Oral Maxillofac Surg 2000; 29:337-340.

5. Su-Gwan K. Treatment of temporomandibular joint ankylosis with temporal muscle and fascia flap. Int J Oral Maxillofac Surg 2001;30:189-193.

6. Dimitrulus G. The interpositional dermis-fat graft in the management of temporomandibular joint ankylosis. Int J Oral Maxillofac Surg 2004;32:360-362.

7. Rowe NL. Ankylosis of temporomandibular joint. J R Coll Surgeon Edin 1982; 27: 67-69.

8. Posnik JC, Goldstein JA. Surgical management of temporomandibular joint ankylosis in pediatric population. Plast Reconstr Surg 1993;91:791-798.

9. Kaban LB, Perrot DH, Fisher K. A protocol for management of TMJ ankylosis. J Oral Maxillofac Surg 1990;48:1145-1151.

10. Roychoudhury A, Prakash HT, Rikha A. Functional restoration by gap arthroplasty in temporomandibular joint ankylosis: a report of 50 cases. Oral Surg Oral Med Oral Pathol Oral Radiol Endod 1999;87:166-169.

11. Molla MR, Shreshtha KR. An analytic study of surgical management of the temporomandibular joint ankylosis: an experience in Bangladesh. Banglad Med Res Counc Bull 1996; 22: 43-50.

12. Ansari SR, Iqbal S, Aslam S. Surgical correction of TMJ ankylosis: a study on the incidence and evaluation of success rates of various surgical procedures. Pak Oral Dent J 2003; 23: 105-112.

13. Chidzonga MM. Temporomandibular joint ankylosis: review of thirty two cases. Br J Oral Maxillofac Surg 1999;37:123-126.

14. Larheim TA. Current trends in temporomandibular joint imaging. Oral Surg Oral Med Oral Path Oral Radiol Endod 1995;80:555-576.

15. Gorgu M, Erdogan B, Akoz T, Kosar U, Dag F. Three dimensional computed tomography in evaluation of ankylosis of the temporomandibular joint. Scand J Plast Reconstr Hand Surg 2000;34:117-120.

16. Toyama M, Kurita k. Koga K, Rivera G. MR arthrography of temporomandibular joint. J Oral Maxillofac Surg 2000;58:979-984.

17. Shah AA. Silastics as interpositional gap arthroplasty in TMJ ankylosis. Ann K E Med Coll 2004;10:84-85.

18. Kazanjian VH. Temporomandibular joint ankylosis. Am J Surg 1955;90:905-908.

19. McCarthy JG, Stelnicki EJ, Grayson BH. Distraction osteogenesis of the mandible: a ten year experience. Semin Orthodontics 1999;5:3-8.

20. Kreutziger KL. Surgery of the temporomandibular joint. Oral Surg 1984; 58; 637-646.

21. Warraich RA. Temporomandibular joint ankylosis - a preventable entity? Ann King Edward Med Coll 2001; 7:168-169. 